\title{
Alkaptonuria: treasure your exceptions
}

\author{
Timothy M. Cox
}

Received: 29 July 2011 /Revised: 29 July 2011 / Accepted: 8 August 2011 / Published online: 22 September 2011

(C) SSIEM and Springer 2011

Archibald Garrod's'Debt of Science to Medicine' (Garrod 1924) still holds and it is only now that signs of repayment are visible. The arduous search for so-called 'disease genes' has always been predicated on the promise of therapeutic innovation for each of the thousands of inherited diseases. In the first hundred years after Garrod's discovery of the inborn errors and despite comprehensive studies of their biochemical genetics, beyond nutritional manipulation and co-factor supplementation, few therapeutic innovations have been brought into clinical practice. Now understood as an authentic disease, and emblematic of countless inborn errors of metabolism, alkaptonuria is a very rare (ultraorphan) condition, and despite all that we know of its biochemistry and molecular genetics, it continues to hover frustratingly on the threshold of innovative therapeutics.

The recent crisis in drug discovery experienced by practically all major pharmaceutical companies, paradoxically gives ground for hope that more courageous investment in informative rare diseases will be forthcoming. There has been a spate of acquisitions by 'Big Pharma' of smaller but successful biotechnology companies: the latter may have creditable scientific ideas and 'discovery pathways', but without the clinical trial expertise and resources fully to develop innovative agents, they remain an empty platform for experimental and translational medicine in the field of rare diseases -'orphans unadopted'. For alkaptonuria, a rich vein of treasure is yet to be found within the

Communicated by: Verena Peters

Competing interest: None declared.

T. M. Cox $(\bowtie)$

University of Cambridge,

Cambridge, UK

e-mail: tmc12@medschl.cam.ac.uk old mine of classical discovery in genetics and biochemistry. Rewarding exploration of this treasure will depend as much on the need to succeed as it will on the contemporary availability of powerful molecular tools, translational scientists and clinicians to investigate the field.

Definitive treatment of alkaptonuria and type I tyrosinaemia, including the clinical use of nitisinone, will come from a thorough understanding of tyrosine metabolism which owes its origin to the classical studies of Garrod and Bateson. It is now clear that successful development of innovative therapy for alkaptonuria will depend on the emerging power of collaborations between patient-based organizations, professional groups and industry. Formidable regulatory (and hence funding) obstacles lie ahead and strong advocacy will be needed to ensure that even the favoured agent, nitisinone, can be evaluated wisely for prophylaxis in the face of an existing failed phase II trial. Long-term studies after early introduction of the drug will be critical to success but are likely to present conceptual challenges for regulators who are unwilling to accept evidence from any other source than the randomized double-blinded cross-over clinical trial. For ultra-orphan diseases, and where blinded control studies are impossible (urinary changes preclude this option in alkaptonuria) unconventional trial designs and long-term observational studies will be of crucial value.

The combination of strong commercial incentives with better informed advocacy on behalf of patients, gives hope that the full potential of orphan drug legislation can be realized. Taking the example of the European Organization for Rare Diseases - a non-governmental patient-driven alliance of patient organizations and individuals - this organ, has with others been instrumental in driving the adoption of the European Council recommendation (2009/C 151/02) for comprehensive National action in the field of rare diseases 
in each constituent country throughout Europe by 2013 . Although these are laudable initiatives, the critical needs of countless patients with innumerable rare diseases remain unmet. Over $95 \%$ of rare diseases lack treatments often when there have been successful therapeutic studies in animal models.

Although there may be plausible treatments available for testing in rare disorders, there is often a failure to utilize the regulations for accelerated approval (which allow the use of surrogate endpoints to achieve marketing approval for a given agent). Such failings have recently been emphasized in stark terms by Brigitta Miyamoto and Emil Kakkis (2011) who also point out that in many rare diseases where there is no precedent for treatment, difficulties arise in gaining acceptance of novel surrogate endpoints. These hurdles are inappropriate and formidable to overcome - especially when combined with the tendency of some personnel in the regulatory agencies not to accept or to facilitate design of unconventional clinical trials often for rare diseases. The introduction of orphan drug legislation has much to celebrate (Haffner 2006; European Committee for Orphan Medicinal Products, 2011) but complacency is unconscionable in the face of the paradigm case of alkaptonuria, the first inborn error of metabolism.

\section{Note}

This editorial is part of a review about the scientific heritage of rare human disorders and the genetic dissection of biochemical pathways.Alkaptonuria and other disorders of aromatic amino acid metabolism have a special place in this legacy. The review opens wider discussion about clinical discovery and therapeutic development for rare disorders through a combination of informed advocacy driven by patients and comprehensive utilization of orphan drug legislation, fully available in JIMD Reports, DOI 10.1007/8904_2011_93.

\section{References}

European Committee for Orphan Medicinal Products (2011) European regulation on orphan medicinal products: 10 years of experience and future perspectives. Nat Rev Drug Discov 10:341-349

Garrod AE (1924) The debt of science to medicine, Harveian Oration, before the Royal College of Physicians on St Luke's Day 1924. 30pp. Clarendon Press, Oxford University

Haffner ME (2006) Adopting orphan drugs-two dozen years of treating rare diseases. N Engl J Med 354:445-447

Miyamoto BE, Kakkis ED (2011) The potential investment impact of improved access to accelerated approval on the development of treatments for low prevalence rare diseases. Orphanet J Rare Dis 6:49 